\title{
O cirurgião cardiovascular como intervencionista
}

\author{
Rui M. S. ALMEIDA
}

\section{'Quando a gente pensa que sabe todas as respostas, vem a vida e muda todas as perguntas'.}

A evolução da cirurgia cardiovascular, nos últimos anos, foi uma grata surpresa, para todos os que acompanham a sua história. O tratamento das doenças tem sido extremamente efetivo e a sua evolução progrediu ao longo dos tempos, para uma melhora dos resultados clínicos, apesar da piora das doenças a serem tratadas, bem como das comorbidades e idade avançada, dos pacientes, quando de seu tratamento cirúrgico. Esta verdade foi uma realidade até ao final do século passado.

A situação começou a ter outra perspectiva, quando Gruentzig, em 1974, realizou a primeira angioplastia coronariana com balão, revolucionando o tratamento da doença arterial coronariana e iniciando um dos mais difundidos métodos de tratamento da atualidade. Onze anos após, Julio Palmaz iniciou o uso de dispositivos endovasculares expandidos por balão, para o tratamento arterial periférico, e Juan Parodi desenvolveu o primeiro dispositivo para aorta, em 1991. Com esta evolução, deu-se início a uma nova subespecialidade, a cirurgia endovascular, que tem crescido de importância nos últimos anos e aumentado a sua área de atuação para diversos locais, sendo que no ano de 2002 se implantou a primeira prótese valvular aórtica através de um procedimento endovascular. Apesar de nova, esta subespecialidade tem sido alvo do interesse de diversas especialidades tradicionais, como a cirurgia cardiovascular, a cardiologia invasiva, a cirurgia vascular e a radiologia intervencionista, todas com interesses próprios, em função da alta tecnologia empregada nestes procedimentos [1]. Com este imenso número de profissionais, e seu interesse comum neste tipo de terapêutica, faz-se necessário avaliar as razões que tornam o cirurgião cardiovascular elegível para realizar procedimentos endovasculares, pois estes, são apenas uma outra forma de tratar doenças historicamente resolvidas por cirurgiões cardiovasculares.

Em primeiro lugar, devemos considerar que a cirurgia cardiovascular está bem organizada e rotinizada, com a maioria de seus tratamentos bem estabelecidos, e uma das mais bem sucedidas especialidades médicas do final do último século. Como em outras especialidades é possível verem-se duas formas de atuação: a convencional, representada por tratamentos conceituados, que podem evoluir na sua forma de realização por pequenas diferenças, como no caso da cirurgia de revascularização do miocárdio e o tipo de solução cardioplégica usada ou o implante de enxertos arteriais; e a inovadora, representada por um novo tipo de tratamento completamente diferente do convencional, e para o qual há quebra dos paradigmas já estabelecidos [2].

Por este motivo, corre-se o risco, de em algumas situações, as cirurgias convencionais tornarem-se obsoletas, ao serem substituídas por procedimentos inovadores, porém estes têm um seguimento menor, e por vezes são realizados por especialista com um treinamento diminuto, se não houver algumas mudanças. O segundo ponto importante é que o aparecimento destas novas formas de tratamento tem levado à solicitação de seu uso por parte dos pacientes, em face de serem realizados com um menor trauma cirúrgico, apresentarem um tempo de internamento menor e integrarem o indivíduo novamente no seu meio mais rapidamente. Outro fator é a indústria que fornece informação diretamente ao consumidor final, o paciente, e incentiva o emprego de seu material, através de uma política agressiva, em função de razões econômicas. Estas informações por vezes são tendenciosas, levando o paciente a expectativas não realísticas.

Com o aumento do número destes procedimentos endovasculares, houve a diminuição do número de procedimentos convencionais realizados, com um importante impacto na esfera econômica dos cirurgiões, e desta sorte nos rumos da especialidade, demonstrado pela diminuição de entrada de médicos nos programas de treinamento para cirurgia cardiovascular.

Para que esta situação não persista, há que promover algumas mudanças não só no treinamento do cirurgião cardiovascular, mas também na mentalidade dos mesmos, para se adaptarem a novas situações $[3,4]$.

Várias são as razões pelas quais a cirurgia cardiovascular deve participar da evolução da forma de tratamento destas doenças. Desde o início da nossa especialidade, aprendemos a realizar o tratamento de uma série de doenças, 
através do que hoje chamamos de cirurgias convencionais, e temos o conhecimento anatômico e fisiopatológico para tal, bem como para realizar o diagnóstico pré-operatório, os cuidados pós-operatórios e o tratamento das possíveis complicações, que possam ocorrer. Para mais ao saber tratar tanto do modo convencional como do endovascular, o cirurgião abre mão do viés que possa existir quando somente se consegue realizar uma forma de tratamento, oferecendo o melhor método terapêutico para o paciente e aplicando com rigor a ciência para também garantir a segurança de seus pacientes.

Abordaremos nesta apresentação alguns aspectos, que achamos ser necessário realizar para mudar os paradigmas de treinamento de cirurgiões cardiovasculares, tanto aqueles em fase de formação, como os que necessitam de ser treinados de novo, agora numa nova área, para os quais há a necessidade de mudança de visão e mentalidade.

Após a decisão de iniciar-se na terapêutica endovascular, o cirurgião deve estar preparado para começar sua caminhada do ponto zero. A curva de aprendizado, para a realização de cirurgias endovasculares, tem seu início numa dedicação á interpretação de exames de imagem, visto que a avaliação e escolha da endoprótese e a orientação tridimensional é a chave para o sucesso do tratamento endovascular. O cirurgião tem de ter um importante domínio de exames como a tomografia computadorizada, para poder interpretar e mensurar imagens de vasos a serem tratados, bem como de ecocardiografia, especialmente a transesofágica, para casos de fechamento de comunicações intracardíacas ou de implante de válvulas cardíacas.

É de extrema importância que o cirurgião saiba interpretar e mensurar as imagens obtidas através destes exames para poder decidir não só da possibilidade de realizar o tratamento endovascular, como também, para poder realizar a solicitação do tipo de prótese a ser usada, em função de uma série de variáveis. No caso de tratamento de aneurismas da aorta, para realizar a indicação de um tratamento endovascular, além da clínica do paciente, há que se levar em consideração a anatomia da aorta, para se poder decidir qual a melhor prótese a ser usada. Diferentes fabricantes possuem próteses com características diferentes e especiais, que podem ou não ser adequadas a determinadas situações. Qual a mais adequada para um determinado caso, depende de uma profunda análise da anatomia e fisiologia do local, no qual será realizado o implante. Este conhecimento é igual ao que gerações anteriores, de cirurgiões cardiovasculares, tinham na interpretação de cineangiografias.

Outra das habilidades que tem de ser aprendida é o do uso de introdutores, fios guias e cateteres, bem como, da manipulação dos mesmos. Este conhecimento baseia-se em saber as características, dos cateteres e sua aplicação, tanto as gerais (tipo de material, tipo de construção, revestimentos, diâmetro interno e externo, comprimento, formato da ponta (“J”, “Cobra”, "Pigtail”) e configuração dos furos, como as específicas (tipo de material da ponta e uso de cateteres centimetrados) em cada território vascular, para prevenir complicações e perda de tempo.

Para tal, deveremos realizar treinamento, em um primeiro tempo, em um laboratório de hemodinâmica, para familiarizarmo-nos com o correto uso da mesa e equipamento de radiologia, bem como de bombas de infusão de contraste; num segundo tempo devemos realizar alguns procedimentos diagnósticos, sob supervisão de um especialista experiente.

Após estes conhecimentos básicos, para se obter habilidade em cirurgia endovascular, devemos fazer uso de modelos de simulação, para iniciar o treinamento propriamente dito. Vários são os modelos de simulação [5], sendo que cada um deles apresenta seus prós e contras:

1) Sintético (vantagens - trabalho estandardizado, barato, facilmente transportável e não há a necessidade do uso de radiologia; desvantagens - não validado como forma de avaliação, uso único de endopróteses, sem reprodução do comportamento dinâmico do sistema arterial)

2) Animal (vantagens - alto grau de realidade, simulação completa; desvantagens - não validado, uso único da endóprotese, diferença de tamanho e anatomia com humanos, problemas éticos e legais, alto custo)

3) Cadáveres Humanos - (vantagens - alto grau de realidade, simulação completa; desvantagens - não validado, uso único da endóprotese, alto custo)

4) Realidade Virtual (vantagens - mudança das especificações do paciente, alto grau de realismo, re-uso do material endovascular; desvantagens - complicado para montar e quebra freqüente)

Dos anteriores aquele que parece ter o melhor resultado, para treinamento, é o da realidade virtual, porém o sintético é o de mais fácil acesso. O tipo de treinamento "hands-on" é essencial para se obter uma habilidade prática efetiva e pode ser realizado com um número significativo de indivíduos sem perda da qualidade de ensino.

Concomitantemente com este treinamento, no caso de cirurgiões cardiovasculares já formados, há a necessidade da frequência de serviços especializados, que tenham um movimento diário significativo de casos endovasculares, e não tão somente a frequência de cursos de reciclagem ou visitas “de final de semana” [6]. Observando como esta nova forma de tratamento é realizada, o cirurgião familiarizase com as indicações e com toda a infra-estrutura necessária para a realização deste tipo de cirurgia. Em seguida, o candidato a cirurgião endovascular deve solicitar que um especialista, já formado nesta terapêutica, o auxilie numa série de cirurgias, até que tenha adquirido todos os 
conhecimentos para a sua execução com desenvoltura. Outro fator que pode ser usado para resolver este problema é a associação com cirurgiões vasculares ou radiologistas intervencionistas para o tratamento endovascular da aorta e seus ramos periféricos e a associação com cardiologistas intervencionistas para o tratamento de doenças cardíacas (comunicações intracardíacas e substituições valvares).

Após este treinamento, e para se obterem resultados de alta qualidade, é necessário o uso de um laboratório, ou sala de cirurgia própria, com um aparelho de hemodinâmica e capaz de realizar cirurgias híbridas, ou seja, a combinação de procedimentos endovasculares com cirurgias convencionais ou abertas. Existe uma dificuldade de entendimento da importância deste ambiente híbrido. Esta área tem historicamente sido utilizada por cardiologistas e radiologistas intervencionistas que são territoriais em relação ao seu uso. Os cirurgiões passaram a ocupar um espaço antes restrito e estes profissionais. Outro impedimento é o dos custos a serem realizados pelos hospitais na aquisição deste equipamento, que pode ser resolvido com a apresentação das vantagens clínicas e econômicas a serem obtidas pelas instituições hospitalares [7].

Em relação aos médicos ingressando na residência de cirurgia cardiovascular, já foi modificado o programa, mas há que se modificar ainda mais a modulação da mesma, de modo que desde o seu início possam freqüentar o laboratório de hemodinâmica e ter contato com este tipo de terapêutica, e acima de tudo, iniciar uma nova fase de aprendizado ao incluir estes procedimentos na rotina da residência.

Paralelamente com todo este esforço individual, há que existir da parte da Sociedade Brasileira de Cirurgia Cardiovascular e de seu departamento especializado, o Departamento de Cirurgia Endovascular, uma atitude não só para tornar possível a atualização científica, como também a de criar formas de treinamento tanto em modelos cirúrgicos, como em centros de treinamento de excelência. A vantagem do uso de modelos de simulação é de poder ter a capacidade não só de iniciar o treinamento cirúrgico, mas também de poder criar situações complicadas e poder desenvolver estratégias que as consigam resolver.

Também e com uma importância extrema o credenciamento deste tipo de tratamento, através do estabelecimento de regras para a sua realização, da confecção de diretrizes para serem seguidas tanto por seus associados como por todas os outros profissionais que realizam estes procedimentos e da avaliação da qualidade deste tratamento, por meio de trabalhos científicos [8].

A participação da indústria, de uma forma independente, também é importante para o apoio na realização dos cursos de treinamento, bem como, no uso de simuladores médicos, visto cada um destes dispositivos, terem características diferentes, não só quanto ao seu posicionamento no local a ser tratado, como também quanto ao mecanismo de instalação dos mesmos. Estes cursos de treinamento devem ser oferecidos, iniciando-se pelos básicos, como os de angiografia diagnóstica e manipulação de cateteres, até aos de intervenções mais especializados como a colocação de endopróteses aórticas, oclusores de comunicações intracardíacas e colocação de válvulas cardíacas.

Apesar do seguimento a longo prazo deste tipo de procedimento não estar ainda perfeitamente definido, as vantagens têm sido evidentes especialmente para a população mais idosa e para aquela com maior número de comorbidades. As informações fornecidas também aos pacientes têm tido um grande peso no aumento tanto das indicações como no aprendizado, por parte dos médicos destes procedimentos. Apesar de ter havido um enorme progresso nesta área nos últimos anos, muito ainda tem de ser realizado para que estes procedimentos não só se estabeleçam definitivamente como melhor forma terapêutica com resultados iguais ou superiores às cirurgias convencionais abertas.

\section{REFERÊNCIAS}

1. Diethrich EB. Future potencial of endovascular techniques for vascular surgeons. Semin Vasc Surg. 2003;16(4):255-61.

2. Cohen DJ. Cardiothoracic surgery at a crossroads: the impact of disruptive technologic change. J Cardiothorac Surg. 2007;2:35.

3. Chan YC, Morales JP, Taylor PR. Training in aortic surgery requires radical change. Eur J Vasc Endovasc Surg. 2007;33(5):516-7.

4. Wheatley GH, Diethrich EB. How to retrain the cardiothoracic surgeon. Interact Cardiovasc Thorac Surg. 2006;5(3):236-7.

5. Neequaye SK, Aggarwal R, Van Herzeele I, Darzi A, Cheshire NJ. Endovascular skills training and assessment. J Vasc Surg. 2007;46(5):1055-64.

6. Kouchoukos NT, Bavaria JE, Coselli JS, De La Torre R, Ikonomidis JS, Karmy-Jones RC, et al. Guidelines for credentialing of practitioners to perform endovascular stentgrafting of the thoracic aorta. Ann Thorac Surg. 2006;81(3):1174-6.

7. Moneta GL, Zierler RE, Zierler BK. Training and credentialing in vascular laboratory diagnosis. Semin Vasc Surg. 2006;19(4):205-9.

8. Almeida RM, Leal JC, Saadi EK, Braile DM, Rocha AS, Volpiani G, et al. Thoracic endovascular aortic repair: a Brazilian experience in 255 patients over a period of 112 months. Interact Cardiovasc Thorac Surg. 2009;8(5):524-8. 